\title{
Désir et sexualité non normatives au Maghreb et dans la diaspora, dossier coordonné par Domingo PUJANTE GONZÁLES
}

\section{Carminella Biondi}

\section{OpenEdition}

\section{Journals}

Édition électronique

URL : https://journals.openedition.org/studifrancesi/12223

DOI : $10.4000 /$ studifrancesi. 12223

ISSN : 2427-5856

Éditeur

Rosenberg \& Sellier

\section{Édition imprimée}

Date de publication : 1 avril 2018

Pagination : 174-175

ISSN : 0039-2944

\section{Référence électronique}

Carminella Biondi, « Désir et sexualité non normatives au Maghreb et dans la diaspora, dossier coordonné par Domingo pujante gonzáles », Studi Francesi [En ligne], 184 (LXII | I) | 2018, mis en ligne le 04 juillet 2018, consulté le 17 novembre 2021. URL : http://journals.openedition.org/studifrancesi/12223 ; DOI : https://doi.org/10.4000/studifrancesi. 12223

Ce document a été généré automatiquement le 17 novembre 2021.

\section{(c)}

Studi Francesi è distribuita con Licenza Creative Commons Attribuzione - Non commerciale - Non opere derivate 4.0 Internazionale. 


\title{
Désir et sexualité non normatives au
} Maghreb et dans la diaspora, dossier coordonné par Domingo PUJANTE GONZÁLES

\author{
Carminella Biondi
}

\section{RÉFÉRENCE}

Désir et sexualité non normatives au Maghreb et dans la diaspora, dossier coordonné par Domingo PUJANTE GONZÁLES, «Expressions maghrébines» 16, n. 1, 2017, 272 pp.

1 Un travail critique consacré à la représentation des sexualités non normatives n'aurait désormais aucun caractère de nouveauté, voire de rupture, s'il ne s'agissait d'une étude concernant un territoire, le Maghreb, où toute «différence» dans le domaine de la sexualité est encore poursuivie par la loi et, plus qu'ailleurs, est cause d'émargination familiale et sociale. Dans son introduction, qui porte le titre du dossier (pp.1-19), Domingo PUJANTE GONZÁLES fait un bilan de la situation socio-politique, du corpus littéraire et artistique, des textes critiques de référence sur le sujet (généraux et spécifiques, car il reconnaît qu'il y a eu quelques pionniers dans ce domaine des études maghrébines), et il indique enfin l'orientation et le but des recherches: «Dans ce dossier, nous avons [...] envisagé d'analyser ce corpus dans sa relation aux revendications politiques et à l'engagement social et associatif, en intégrant [...] des notions propres aux études culturelles, postcoloniales et à la théorie queer. Nous avons également suggéré aux auteurs de porter une attention toute particulière à des aspects liés au renouveau des récits autobiographiques, à l'appropriation et à l'exploration du corps transidentitaire, aux espaces ou à la topographie du désir non normatif, au renversement des rapports hiérarchiques des sexes, à l'influence du religieux et aux effets croisés ou à l'intersectionnalité des rapports ethniques, de classe, de genre et de 
sexualité» (p. 15). On ne saurait synthétiser de manière plus efficace le contenu du dossier qui, pour une fois, correspond dans une large mesure aux attentes qu'il suscite, car il y a de tout cela dans les onze articles qui le composent et qui réussissent à rendre tangibles les convergences et les jeux complexes qui situent une vie hors norme dans un contexte socio-politico-religieux et les stratégies créatives mises en acte pour leur transfert dans une œuvre, que ce soit une œuvre littéraire, sculpturale ou filmique.

2 Le dossier est divisé en deux parties: la première, la plus longue, s'occupe de la «création littéraire» (pp.23-148), la deuxième de la «création artistique et cinématographique» (pp.149-216). Mais il y a un fil rouge qui parcourt tous les textes et contribue à l'unité du recueil et, ajouterai-je, à son intérêt: il ne s'agit pas d'y étudier tout simplement des objets littéraires et artistiques qui se limitent à raconter une histoire de «diversité» sexuelle, mais plutôt d'ouvrages qui font émerger les tourments d'une réalité complexe, plurielle, incertaine, où la fragilité des corps s'associe à la fragilité d'âmes qui se cherchent et qui ne trouvent dans leur milieu aucun support car surtout l'homosexualité masculine - mais féminine aussi - mine les bases d'une société patriarcale, dont la force de l'homme, ou du moins, son apparence constituent la structure portante, aussi bien que la soumission de la femme. La première partie s'ouvre sur un article de Khalid zEKRI qui pose les bases «théoriques» du sujet: Le "genre» en littérature maghrébine et arabe: Une déconstruction productive (pp. 23-22). L'auteur y indique aussi les textes critiques de référence utilisés par presque tous les collaborateurs, en particulier l'ouvrage, défini «fondateur», de Judith Butler, Trouble dans le genre (2005), traduction française d'un essai paru en langue originale, aux ÉtatsUnis, en 1990. Suivent des contributions focalisées sur quelques-uns des auteurs qui ont le plus contribué à l'affirmation d'une littérature de rupture: Jean ZAGANIARIS (un critique qui est aussi romancier), Fragilité des corps, vulnérabilité des masculinités: Représentations des pratiques sexuelles non normatives dans les romans de Mokhtar Chaoui et Youssef Wahboun (pp. 35-52); Hervé SANson, Sexualité, désir et genre dans les fictions de Mohamed Leftah (pp. 53-67); Kirsten HUSUNG, «Combien de temps pour devenir une femme?». Sujétion et transgression chez Nina Bouraoui (pp. 69-83): c'est le seul travail consacré à une écrivaine dans le dossier. Les trois derniers articles de la première partie portent sur l'œuvre de l'écrivain et cinéaste marocain Abdellah Taïa, un musulman qui a déclaré publiquement son homosexualité et qui s'est engagé dans une lutte acharnée contre tous les préjugés. Un texte de lui, qui clôt la première partie, raconte sa difficulté d'être et la pénible conquête de soi: "Quarante ans pour pouvoir dire enfin, soulagé, léger, peut-être libre: JE SUIS L'HOMME DE MES DÉSIRS» (Trente, pp. 143-148, explicit). Les articles qui le concernent essaient de cerner tous les aspects de son œuvre: Ralph HEYNDELS, Configurations et transferts de la sexualité, du genre et du désir dans l'ouverture d'“Une mélancolie arabe" d'Abdellah Taïa, ou «le dépassement des frontières» (pp. 85-105); Tina DRANSFELDT CHRISTENSEN, Breaking the Silence: Between Literary Representation and LGBT, Abdellah Taïa as Author and Activist (pp. 107-125): la critique s'appuie en particulier sur un texte de Taïa dont le titre est en même temps une provocation et un acte de bonne volonté: L'Homosexualité expliquée à ma mère (in «TelQuel» 367, en ligne). Le dernier article, d'Ismael NAVARRO SOLERA, insiste sur la fonction du corps dans l'acte libertaire et provocateur d'une écriture qui brise finalement le carcan suffoquant des impositions socio-familiales et religieuses: La transgression poétique et politique par le biais du corps dans l'œuvre autofictionnelle d'Abdellah Taïa (pp. 127-141). 
Le premier article de la deuxième partie, de Jesús MARTínez oliva, présente l'œuvre de l'artiste franco-marocain, Mehdi-Georges Lahlou, «Ceci n'est pas une femme musulmane»: Mehdi-Georges Lahlou's Daring Defiance of Gender, Religious, and Cultural Identities (pp. 151-168), tandis que les trois autres s'intéressent à la cinématographie de rupture. Là aussi, comme dans la section précédente, il n'y a qu'une seule femme présente (ce déséquilibre est-il le miroir de la réalité maghrébine, où la femme «non homologuée» aurait plus de difficulté à se dire?), dans l'essai d'Ana MONLÉON DOMínGUEZ, Représentation des sexualités non normatives dans les films de fiction de Nadia El Fani (pp. 169-182); en clôture de la section nous trouvons les essais de Madeleine LöNING, Un film qui «trouble»: Subversion des identités de genre et de la sexualité dans "Much loved" de Nabil Ayouch (pp. 183-197) et d'Aziza Azzouz, Le désir interdit dans "L'Homme de cendres" de Nouri Bouzid: Une dialectique spatio-corporelle du silence (pp. 201-216). Les deux films abordent des thèmes tabous: le premier celui de la prostitution au Maroc, le second celui du viol et des déviances qu'il provoque dans les sujets qui en sont les victimes, qui deviennent aussi les victimes d'une société qui les rejette: «Nouri Bouzid met ainsi l'accent sur l'importance du regard des autres dans la formation d'une identité de l'individu et accuse les autres d'être à l'origine de la claustration des deux protagonistes» (p. 213). Un jugement qui pourrait bien s'appliquer à la majorité des écrivains et des artistes étudiés dans le dossier, des écrivains et des artistes qui ont eu le courage de dire «l'indicible», sous le regard hostile de leur milieu.

4 Le numéro se termine par une section de «Varia», dont les deux articles qui la composent ont quelque consonance avec ceux du dossier, non pas dans le domaine de la sexualité, mais dans celui de la différence: Carla CALARGÉ et Alexandra GUEYDAN-TUREK, Des féminismes du Sud dans Bilqiss de Saphia Azzeddine (pp. 219-235); Nancy ARENBERG, The Effacement of Jewish Identity in Marlène Amar's “La Femme en tête" (pp. 237-250). 